\title{
Risco de Lesões Músculo Esqueléticas nos Enfermeiros: Contributos do Photovoice
}

\section{Maria Helena Presado ${ }^{1}$, Armando David Sousa ${ }^{2}$, Cristina Lavareda Baixinho $^{3}$, Ana Leonor Mineiro ${ }^{4}$, Fátima Mendes Marques ${ }^{3}$, Mário Cardoso $^{1}$ e Tiago Nascimento ${ }^{5}$}

${ }^{1}$ Departamento de Saúde Materna e Obstétrica, Escola Superior de Enfermagem de Lisboa Portugal | mhpresado@esel.pt; mmcardoso@esel.pt | https://orcid.org/ 0000-0002-68527875; https://orcid.org/ 0000-0003-4961-9026

${ }^{2}$ Escola Superior de Enfermagem São José de Cluny, Madeira, Portugal armandodav@gmail.com | https://orcid.org/ 0000-0002-6393-5896

${ }^{3}$ Departamento de Enfermagem de Reabilitação, Escola Superior de Enfermagem de Lisboa, Portugal | crbaixinho@esel.pt; fmarques@esel.pt | https://orcid.org/0000-0001-74171732; https://orcid.org/ 0000-0001-6581-6711

${ }^{4}$ Hospital Garcia de Orta, Almada, Portugal | leonor1981@yahoo.com.br | https://orcid.org/ 0000-0003-4505-6262

${ }^{5}$ Departamento de Administração em Enfermagem, Escola Superior de Enfermagem de Lisboa, Portugal | tnascimento@esel.pt | https://orcid.org/0000-0003-3646-9057

Resumo: Um dos principais problemas de saúde ocupacional dos enfermeiros são as lesões músculo-esqueléticas relacionadas com as condicões de trabalho (LMELT), as atividades específicas dos Enfermeiros e fatores de risco individuais, psicossociais e organizacionais. Objetivos: Analisar a perceção dos estudantes de mestrado sobre o risco de lesões músculo esqueléticas ligadas ao trabalho na sua prática clínica. Métodos: Estudo qualitativo, descritivo e exploratório com recurso ao Photovoice como método de investigação de ação participativa suportado na compreensão das experiências dos estudantes. A análise de conteúdo seguiu as etapas definidas por Bardin e foi suportada pelo software webQDA ${ }^{\circledR}$; Resultados: Os participantes são 6 estudantes da Unidade Curricular Biomecânica no cuidar do Enfermeiro Especialista de Saúde Materna e Obstetrícia. Das 185 unidades de enumeração obtidas, 119 correspondem a fatores de risco físico, 38 a fatores psicossociais e organizacionais e 28 a fatores de risco individual. Sabem os princípios da biomecânica, mas identificam dificuldades na sua utilização face à emergência e natureza da atividade profissional. Conclusões: Salientamos o desadequado uso de espaços e equipamentos face às características individuais que contribuem para o risco dos profissionais no contexto de trabalho, agravadas pela situação de pandemia. Foi essencial a utilização do Photovoice como método de recolha de dados, por possibilitar aceder à perspetiva dos participantes sobre os seus fatores de risco de LMELT e promover um papel mais ativo na sua prevenção.

Palavras-chave: Transtornos Traumáticos Cumulativos; Riscos Ocupacionais; Enfermeiro; Fatores de Risco; Perceção.

\section{Risk of Skeletal Muscle Injury in Nurses: Contributions of Photovoice}

Abstract: One of the nurse's main occupational health problems are the Work-Related Musculoskeletal Disorders (WRMD). There are specific risks related to the nursing activity, as well as individual, psychosocial and organizational risks. Objectives: To analyze the perception of master's students about the risk of musculoskeletal injuries related to work in their clinical practice. Methods: Qualitative, descriptive and exploratory study using Photovoice as a method of investigating participatory action based on the understanding of students' experiences. The content analysis followed the steps defined by Bardin and was supported by the WebQdA ${ }^{\circledR}$ software; Results: The participants are 6 students from the Biomechanical Curricular Unit in the care of the Specialist Nurse, Maternal Health and Obstetrics. Of the 185 enumeration units obtained, 119 correspond to physical risk factors, 38 to psychosocial and organizational factors and 28 to individual risk factors. They know the principles of biomechanics, but they identify difficulties in their use in view of the emergence and nature of professional activity. Conclusions. We emphasize the inappropriate use of spaces and equipment in view of the individual characteristics that contribute to the risk of professionals in the work context, aggravated by the pandemic situation. The use of Photovoice as a method of data collection was essential, as it made it possible to access the perspective of participants on their risk factors for WRMD and promote a more active role in its prevention.

Keywords: Cumulative Trauma Disorders; Occupational Risks; Nurses; Risk Factors: Perception. 


\section{Introdução}

As lesões músculo-esqueléticas ligadas ao trabalho (LMELT) são identificadas como um dos principais problemas globais de Saúde Ocupacional, verificando-se elevado impacto nos Enfermeiros, com prevalências a 12 meses que podem oscilar de $34 \%$ a $88 \%$ (Thinkhamrop et al., 2017). As LMELT podem ser definidas como transtornos traumáticos cumulativos decorrentes de distúrbios isolados ou combinados, nos músculos, articulações, cartilagem ou discos vertebrais, caracterizadas por desconforto, incapacidade ou dor persistente, causada ou agravada maioritariamente pelo desenvolvimento do trabalho e os efeitos do ambiente no qual este trabalho é desempenhado (Boakye et al., 2018).

Os enfermeiros no seu contexto clínico, acarretam elevada exposição a fatores de risco, proveniente dos movimentos repetidos, das posturas inapropriadas, da aplicação de força e pelas vibrações de algumas tarefas. A exigência física e articular de algumas tarefas são extremas, com maior incidência na coluna vertebral. Os Enfermeiros consignam esta exigência em intervenções autónomas e interdependentes como a administração de terapêutica, prestação de cuidados de higiene, posicionamentos, entre outros (Presado et al., 2015; Serranheira et al., 2012).

Na prestação dos Enfermeiros Obstetras, surgem riscos adicionais de acordo com três dimensões percecionadas: o equipamento e os recursos materiais, fatores relacionados com a parturiente e fatores associados com a natureza da tarefa (Presado et al., 2015). Contribuem também para o agravamento das lesões fatores como idade, anos de prática, índice de massa corporal, horas de trabalho, satisfação e stress laboral (Okuyucu et al., 2019).

Atualmente num contexto de pandemia (COVID-19), os enfermeiros são sujeitos à tensão e exaustão física da prestação de cuidados, com dinâmicas de trabalho variadas, oriundas do número crescente de pacientes, com possível deterioração rápida, sujeitos a exposição à doença e à morte (Chang et al., 2020). Embora o stresse, ansiedade e a depressão possam ser vistos como reações emocionais normais diante de uma pandemia (Liu et al. 2020), o seu surgimento e manutenção agravam as LMELT.

Embora os riscos das LMELT já tenham sido identificados desde o século XVIII, a sua desvalorização continua presente, os profissionais e os gestores valorizam a qualidade dos cuidados prestados e desvalorizam a importância da saúde dos prestadores, havendo mesmo a possibilidade de ocorrer stress pós-traumático (Bianchi et al., 2019).

A desvalorização destes fatores em prol das necessidades dos doentes, da resiliência dos profissionais, das necessidade económica e sociais, potenciam o agravamento das lesões na dimensão temporal contribuindo para a ocorrência do presenteísmo - entendido como o exercício de funções no local de trabalho em condições de saúde precárias e muitas vezes com um desempenho abaixo do expectável (Rainbow \& Steege, 2017), e consequentemente, no absentismo laboral, com consequências inequívocas na qualidade dos cuidados e com impacto financeiro na sustentabilidade e eficiência das organizações (Aysun \& Bayram, 2017; Luan et al., 2018; Nascimento \& Pestana, 2019). A análise e mitigação dos riscos na prática do Enfermeiro, promovendo o "awareness" dos profissionais, tem um impacto positivo na redução da sintomatologia músculo-esquelética e associado à adequada gestão, promovem o bem-estar do colaborador, a segurança dos pacientes, bem como as dotações seguras nos contextos de prática clínica (Okuyucu et al., 2021).

Com este estudo pretendemos analisar a perceção dos estudantes de mestrado em saúde materna e obstétrica sobre o risco de LMELT na sua prática clínica, decorrente da Unidade Curricular (UC) de Opção: Biomecânica no Cuidar do Enfermeiro Especialista de Saúde Materna e Obstetrícia (UC-BCEESMO). 


\section{Metodologia}

Estudo de abordagem qualitativa, descritivo e exploratório baseado num conhecimento compreensivo cuja interpretação se assumiu como elemento fulcral na investigação. A procura de experiências de ensino-aprendizagem indo ao encontro da realidade do contexto clínico e fomentando o pensamento crítico dos estudantes (Andina-Díaz, 2020; Gonçalves et al., 2021), levou os sete investigadores e docentes da UC-BCEESMO, a lançar o desafio da utilização do Photovoice. Este é um método participativo da metodologia investigativa envolvendo fotografia e reflexão (Wang \& Burris, 1997; Werremeyer et al., 2020). Como prática de produção de conhecimento, o Photovoice assenta em três objetivos: 1) habilitar as pessoas na identificação, registo e reflexão sobre potencialidades e problemas da sua comunidade; 2) promover o diálogo crítico e o conhecimento sobre temas importantes através de grupos de discussão das fotografias; 3 ) dar visibilidade às suas vidas, nomeadamente, a decisores políticos e financeiros (Wang \& Burris, 1997; Wang et al., 1998).

Esta estratégia de investigação de ação participativa é cada vez mais valorizada na área da saúde (Capewell et al., 2020; Santos et al., 2018; Werremeyer et al., 2020) e na formação em enfermagem (Andina-Díaz, 2020), pelo desenvolvimento de competências de comunicação e de intervenção. Deste modo, para responder à questão de investigação: Qual a percepção dos estudantes sobre o risco de LMELT na sua prática clínica? fo solicitado aos estudantes que documentassem a realidade do seu local de trabalho, relativamente ao risco de LMELT. Nesta análise, é impossível distinguir opiniões, perceções e significados individuais desagregados do seu contexto (Baixinho, C.L., Presado, M.H. \& Ribeiro, J., 2019).

As fotos foram obtidas através de máquinas fotográficas, telefones portáteis com câmaras acopladas ou tablets e respeitando o anonimato das pessoas que apareceram nas fotografias (rostos pixelados) (Andina-Díaz, 2020; Santos et al., 2018). Os participantes produziram um texto com uma análise e descrição reflexiva das fotos contextualizadas na problemática das LMELT. Ao partilharem e analisarem as suas fotografias, os estudantes recorreram ao poder da imagem visual para comunicar as suas experiências, perícia e conhecimentos (Wang et al., 1998).

A amostra foi constituída inicialmente por 6 estudantes que aceitaram participar após explicação e esclarecimento de dúvidas sobre o estudo. Os critérios de seleção dos estudantes foram: a) estarem a frequentar a UC BCEESMO e, b) exercerem atividade profissional em contexto de cuidados de saúde.

Após a recolha de dados procedeu-se à análise que envolveu a organização sistematizada dos documentos elaborados pelos estudantes. A constituição de um corpus implicou atender à representatividade, exaustividade, homogeneidade e relevância dos documentos disponíveis em relação ao objeto de estudo (Bardin, 2016).

Os dados foram submetidos a análise de conteúdo (Bardin, 2016), suportada pelo software WebQdA ${ }^{\circledR}$ (Costa \& Amado, 2018), por possibilitar a partilha de informação entre investigadores, a análise de dados não estruturados, como imagens (Freitas et al., 2017).

Os dados foram transformados constantemente e agregados em unidades temáticas proporcionando uma descrição exata das caraterísticas pertinentes do conteúdo (Bardin, 2016). Posteriormente, realizou-se a categorização e a contagem frequencial, que incidiu no tipo de unidades de registo (UR). Embora os programas de análise qualitativa sejam uma ferramenta facilitadora, estes não substituem a codificação humana (Fornari \& Fonseca, 2020). Dois investigadores asseguraram a atribuição dos códigos aos discursos, sendo validada pela restante equipa de investigadores, atendendo ao carácter colaborativo do WebQdA ${ }^{\circledR}$ (Costa \& Amado, 2018). 
A Comissão de Ética da ESEL aprovou previamente a investigação e foi solicitado aos estudantes o consentimento informado. A confidencialidade foi assegurada tendo sido garantido $\mathrm{o}$ anonimato dos participantes, os nomes dos estudantes foram identificados com um nome fictício, criando um código $(\mathrm{P})$ que será utilizado neste trabalho. Apenas a equipa de investigadores teve acesso aos projetos de intervenção.

\section{Resultados}

Participaram no estudo 6 estudantes do sexo feminino, que frequentaram a UCBCEESMO. A idade dos participantes varia entre os 28 anos e 48 anos com uma média de 34,5 anos, o tempo profissional como enfermeiro varia entre 6 anos e 27 anos (média de 11,3 anos) e o tempo de exercício de funções no serviço atual varia entre 2 e 10 anos ( 6,8 anos de tempo médio). Relativamente às características pessoais dos participantes, o peso varia entre $50 \mathrm{Kg}$ e $65 \mathrm{~kg}$ (média de $58,7 \mathrm{Kg}$ ), a altura entre $1,62 \mathrm{~m}$ e $1,80 \mathrm{~m}(1,7 \mathrm{~m})$ e o IMC varia entre 19,1 e 23,9 (média de 21), encontrando-se dois enfermeiros em situação de baixo peso.

Dos textos produzidos pelos participantes com base nos registos fotográficos, foi realizada a análise qualitativa recorrendo ao WebQDA ${ }^{\circledR}$. Na pesquisa das palavras mais frequentes, obtivemos a palavra "trabalho" com 31 referências, seguindo-se a "posição" com 24 referências e as palavras "serviço" e "altura" ambas com 19 referências (Figura 1).

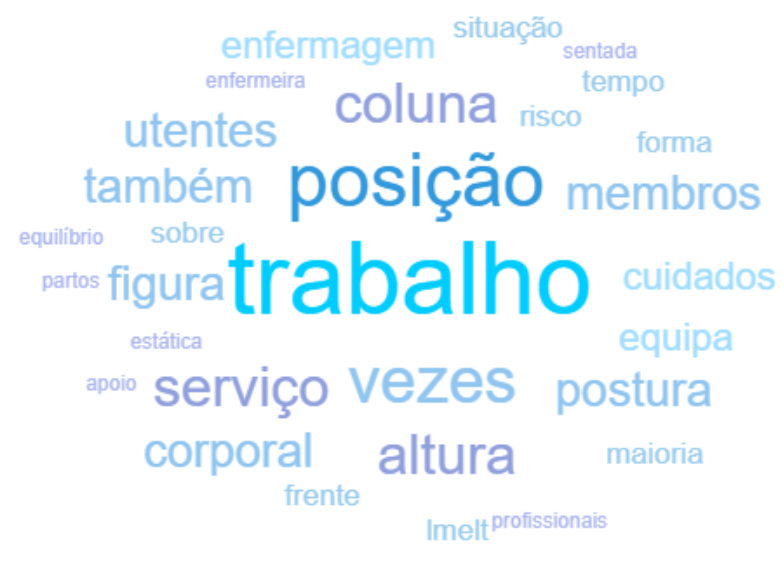

Fig. 1. Palavras mais frequentes dos textos narrativos, Lisboa, 2020.

O resultado da codificação dos textos permite-nos verificar que, das 185 unidades de enumeração (referencias) obtidas: 119 correspondem aos fatores de risco físico, 38 aos fatores de risco psicossociais e organizacionais e, 28 aos fatores de risco individual (Tabela 1).

Tabela 1. Referenciação dos fatores de risco, Lisboa, 2020.

\begin{tabular}{|c|c|c|}
\hline Dimensão dos Fatores de Risco & Ref & Fontes \\
\hline Físico & 119 & $1,2,3,4,5$ e 6 \\
\hline Psicossociais e Organizacionais & 38 & $1,2,3,4,5$ e 6 \\
\hline Individual & 28 & $1,2,3,4,5$ e 6 \\
\hline Total & 185 & 6 \\
\hline
\end{tabular}


Os participantes identificaram como Fatores de risco Psicossociais e Organizacionais, as características físicas dos espaços, ausência de equipamentos ou inadequados e a dinâmica da instituição/serviços (trabalho por turnos, rácios e sobrecarga de trabalho), expressas nos verbatins:

- “(...) o espaço físico dos quartos é reduzido, para além das camas, mesas de cabeceira e mesas de apoio à refeição, ainda é necessário criar um espaço com materiais de consumo clínico" (P1).

- “(...) contexto laboral é constante a sobrecarga e stress de trabalho, pois é um serviço de enorme afluência de utentes na sua maioria totalmente dependentes e que frequentemente necessitam de cuidados especializados, existindo escassos recursos humanos para dar resposta a estas necessidades" (P4).

- "(...) serviço dinâmico, com poucas rotinas estabelecidas e por isso com elevada imprevisibilidade dos turnos, o que conduz a um permanente estado de alerta" (P3).

- "(...) existe um transfere, que se encontra no bloco operatório, que se localiza num dos extremos arquitetónicos do serviço, conduzindo a que este apenas seja utilizado maioritariamente nesta localização" (P3).

- “(...) trabalha por turnos, com elevadas cargas de horário, realizando muitas vezes dois e três turnos noturnos por semana, devido à escassez de recursos humanos" (P3).

- “(...) as pausas laborais, sejam consecutivamente adiadas e muitas vezes não realizadas, resultando numa sobrecarga permanente da equipa de enfermagem devido aos ritmos intensos de trabalho" (P3).

- “(...) no início da pandemia cada quarto tinha uma lotação máxima de dois utentes, atualmente e dado o aumento do número de casos positivos, os utentes com covid positivo, chegam a estar três por quarto" (P1).

O código de árvore do software webQDA ${ }^{\circledR}$ dos Fatores de risco Físico, permite-nos constatar que existem 119 referências relativas à categoria mecânica corporal. Todos os participantes aludiram a aspetos que se enquadram no alinhamento corporal, equilíbrio, movimento corporal, fricção e atrito, força mecânica.

$\mathrm{Na}$ subcategoria alinhamento corporal foi possível verificar que, das 31 referências, 6 mantinham alinhamento, e 25 não mantinham, visível nesta citação: "posição estática flexão da coluna, ocorrendo desequilíbrio corporal, pelo desalinhamento os ombros relativamente às cristas ilíacas, com rotação externa das tíbias e ligeira flexão dos joelhos, para preservar algum equilíbrio" (P5).

O equilíbrio obteve 30 referencias, das quais 27 são de postura em desequilíbrio: "peso não está distribuído uniformemente, sendo que o centro de gravidade se encontra deslocado para a frente, fora da base de sustentação, colaborando para a diminuição do equilíbrio" e "apoiada praticamente num só pé e utilizo a distensão da coluna vertebral de forma a chegar à prateleira mais alta do armário de stock" (P5).

O movimento corporal conseguiu 30 referências, 28 fazem alusão à posição estática por longos períodos, visível nestas citações "(...) a enfermeira encontra-se numa posição estática por um longo período (superior a 6 minutos)" e "(...) ficar em pé e trabalhar nos computadores que estão alocados aos carros de medicação" (P6).

Relativamente a "Fricção e Atrito", apenas apuramos 7 referencias de 5 participantes (fontes). A força mecânica foi aludida por 6 fontes com 14 referencias. 
Classificado como "outros" fatores, encontramos 7 referencias mencionados por 3 participantes, correspondendo à sintomatologia das LMELT, como presente nesta citação: "(...) sintomatologia tende a piorar no fim do turno e quando trabalho por dias consecutivos, e por sua vez, melhora nos dias de folga ou durante as férias" (P4) e a consciencialização da adoção de posturas incorretas: "adoção de posturas inadequadas e viciosas quando estou a exercer a minha prática clínica, pois tenho plena consciência que estou a ser incorreta comigo própria" e "maioria das vezes é sob stresse e de forma rápida que as realizo e não penso nas consequências da forma como as concretizo" (P4).

Tabela 2. Categorização dos fatores de risco físico, Lisboa, 2020.

\begin{tabular}{|c|c|c|}
\hline Fatores de Risco Físico & Ref & Fonte \\
\hline Alinhamento corporal & 31 & 6 \\
\hline Equilíbrio & 30 & 6 \\
\hline Movimento corporal & 30 & 6 \\
\hline Fricção e atrito & 7 & 5 \\
\hline Força mecânica & 14 & 6 \\
\hline Outros & 7 & 3 \\
\hline Total & 119 & 6 \\
\hline
\end{tabular}

No que concerne aos riscos individuais, salientamos que 5 fontes apresentaram 28 referencias às características pessoais como a idade, peso, altura e hábitos de vida como a alimentação e exercício físico.

\section{Discussão}

O Photovoice foi relevante como método de recolha de dados por traduzir a auto perceção dos participantes e permitir uma melhor compreensão do significado das LMELT e, concomitantemente, promover um papel mais ativo na sua prevenção (Topcu et al., 2020).

Os resultados reforçam o que está descrito em outros estudos que identificam um conjunto de riscos associados à prática clínica dos enfermeiros: 1) físico (movimento corporal, alinhamento corporal, equilíbrio, força, e atrito e friç̧ão); 2) individual (idade, peso, altura) e; 3) psicossociais e organizacionais (espaço de trabalho, equipamentos e dinâmica organizacional) (Baixinho, Presado, Marques, \& Cardoso, 2016a).

A organização de um turno de trabalho e a tomada de decisão sobre a adoção dos princípios da biomecânica é complexa e pode ser relevada para segundo plano pela existência de vários focos de atenção, com a obrigatoriedade de rapidamente se passar de uma posição estática para uma dinâmica (Baixinho, Presado, Marques, \& Cardoso, 2016b). Na análise da nuvem de palavras as que emergem com maior expressividade são trabalho e posição, o que de algum modo relaciona bem as dificuldades associadas ao volume de trabalho, diversidade de atividades e alterações frequentes de posição.

O risco acrescido de lesões está associado a períodos prolongados com posturas incorretas, sem respeito pelo alinhamento corporal, mantendo-se na posição de pé com flexão acentuada do pescoço e da região dorsolombar e dorsiflexão quando manipulam "cargas", mantendo simultaneamente movimentos repetitivos, com aplicação de força e elevação do membro superior a 90으 (Ganer, 2016).

$\mathrm{O}$ atual contexto de pandemia patente nas descrições dos participantes, obriga à restruturação dos serviços e um aumento do fluxo de trabalho, bem como, a sobre carga física, emocional e psicossocial, fruto da incerteza diária (Chang et al., 2020; Liu et al., 2020). 
A natureza especifica de algumas atividades desempenhadas pelos enfermeiros (exemplo o parto) têm riscos associados aos movimentos bruscos de pronosupinação, realizados com as mãos elevadas em relação aos ombros (Taghinejad, Azadi, Suhrabi \& Sayedinia, 2016), que dificilmente podem ser controlados. Assim, uma atividade que já é por si 'penosa' torna-se difícil de controlar por um conjunto de fatores profissionais, psicossociais e organizacionais.

Os resultados desta pesquisa reforçam o supracitado, com as participantes a descrever situações em que não conseguem manter o equilíbrio, com má distribuição do seu peso corporal e não manutenção do centro de equilíbrio na base de sustentação. Outras registam os elevados períodos na posição de pé, a fricção e o atrito na execução das atividades e a força também como elementos de difícil controlo, o que vai ao encontro dos outros resultados obtidos também por abordagens qualitativas (Long, Bogossian \& Johnston, 2013; Ganer, 2016).

Estes riscos que advém da tomada de decisão das profissionais, e da falta de formação, são agravados pelo ambiente de trabalho inadequado, materiais e equipamentos desajustados, às suas características antropométricas e às exigências tempo e rácio profissional/utente (Baixinho et al., 2016a; 2016b). Corroborando o que outros autores advogam sobre a necessidade de reforçar a formação ao nível pré e pós-graduada sobre a biomecânica (Presado et al., 2015), os resultados apontam que mesmo com formação há dificuldades em manter a segurança postural, o que justifica uma aposta em políticas de saúde ocupacional que promovam o bem-estar e controlem os fatores de risco com participação ativa de todos os envolvidos.

Este repto à prevenção justifica-se na elevada prevalência e nas consequências que estas lesões têm para os enfermeiros, para as organizações com o absentismo, e para o sistema de saúde, dado que muitas destas lesões evoluem para a cronicidade e exigem cuidados de reabilitação e cirurgias que aumentam os custos com o tratamento (Aysun \& Bayram, 2017; Boakye et al., 2018; Luan et al., 2018; Nascimento \& Pestana, 2019).

A solução pode passar pela formação contínua em contexto de trabalho, com envolvimento da equipa multiprofissional e recurso à prática simulada de alta-fidelidade (Presado et al., 2015; Baixinho et al., 2016a; 2016b; Baixinho, C.L., Presado, M.H. \& Ribeiro, J., 2019).

Face à gravidade do fenómeno, recomenda-se o desenho de uma intervenção multidimensional para o controlo deste grave problema de saúde pública.

\section{Conclusões}

A análise do resultados tornou evidente os múltiplos fatores de risco de LMELT percecionados pelos participantes na sua prática clínica e as inúmeras exigências biomecânicas da sua atividade, condicionando a adoção frequente de posturas inadequadas, evidenciadas por: adoção maioritária de posições estáticas; posturas forçadas que exigem alinhamentos não naturais do corpo, com maior sobrecarga nas regiões cervical e dorsolombar; desvios do centro de gravidade, com uma base de sustentação pouco ampla, contribuindo para a diminuição do equilíbrio; realização de movimentos repetitivos e; utilização incorreta das alavancas para efetuar força.

Todos os participantes apresentaram fatores de risco individuais; psico-sociais e organizacionais e físicos para o desenvolvimento de LMELT. Neste âmbito, a intervenção ergonómica é imperiosa de modo a minimizar os fatores de risco de LMELT, promovendo a adoção de posturas adequadas por parte dos enfermeiros, de forma a realizarem a tarefa com conforto, segurança e eficiência.

Consideramos importante o controlo do ambiente físico, a organização adequada do espaço de trabalho, a existência de equipamentos funcionais, ergonomicamente concebidos, adaptados e adequados à prática clínica, assim como, um investimento na formação dos profissionais para a prevenção das LMELT, não só em contexto laboral, mas igualmente nos contextos de formação graduada e pós-graduada. 
Este estudo obteve um contributo essencial da Investigação Qualitativa para a compreensão da problemática em análise. A utilização do Photovoice, como método de recolha de dados, na investigação qualitativa, foi essencial aos investigadores, por possibilitar aceder à perspetiva dos participantes e melhor compreender qual a sua perceção acerca dos fatores de risco de LMELT presentes na sua prática clínica e de que forma podem desenvolver estratégias interventivas para prevenção das LMELT. Este estudo permitiu alcançar uma nova visão do fenómeno, recorrendo à perspetiva dos seis enfermeiros participantes, o que favorece a compreensão das LMELT.

Consideramos que este estudo traz contributos relevantes para a prática clínica e para a investigação de natureza interpretativa, quer pelo processo metodológico, quer pelos resultados obtidos.

O enfoque na segurança dos profissionais é essencial, de forma a garantir cuidados de saúde mais seguros, eficientes e produtivos, diminuindo assim o presenteísmo, o absentismo laboral e os gastos com a saúde provocados pelas LMELT.

\section{Referências}

Andina-Díaz, E. (2020). Using photovoice to stimulate critical thinking: An exploratory study with nursing students. Em Revista Latino-Americana de Enfermagem (Vol. 28, pp. 1-7). scielo. https://doi.org/10.1590/1518-8345.3625.3314

Aysun, K., \& Bayram, Ş. (2017). Determining the level and cost of sickness presenteeism among hospital staff in Turkey. International Journal of Occupational Safety and Ergonomics, 23(4), 501-509. https://doi.org/10.1080/10803548.2016.1274159

Baixinho, C. L., Presado, H., Marques, F. M., \& Cardoso, M. (2016a). A segurança biomecânica na prática clínica dos enfermeiros de saúde materna e obstetrícia. Revista Brasileira Promoção da Saúde, 29(sup), 36-43. https://doi.org/10.5020/18061230.2016.sup.p36

Baixinho, C. L., Presado, H., Marques, F. M., \& Cardoso, M. (2016b). Prevenção das lesões músculo-esqueléticas: relatos dos enfermeiros especialistas em saúde materna e obstétrica. Atas CIAIQ, 2, 488-97

Baixinho, C.L., \& Presado, M.H., Ribeiro, J. (2019). Qualitative research and the transformation of public health. Ciência \& Saúde Coletiva; 24 (5)1583-1583. https://doi.org/10.1590/141381232018245.05962019

Bardin, L. (2016). Análise de Conteúdo. Em Edições 70.

Bianchi, T., Belingheri, M., Nespoli, A., De Vito, G., \& Riva, M. A. (2019). Occupational risks in midwifery: From Bernardino Ramazzini to modern times. Safety and Health at Work, 10(2), 245-247. https://doi.org/https://doi.org/10.1016/j.shaw.2018.11.002

Biggs, J., \& Tang, C. (2011). Teaching for quality learning at university (4th ed.). Open University Press. http://books.google.se/books/about/Teaching_for_Quality_Learning_at_Univers.html?id=XhjR BrDAESkC\&pgis $=1$

Boakye, H., Numarce, B., Ameh, J. O., \& Bello, A. I. (2018). Work-related musculoskeletal disorders among nurses and midwives at a municipal health facility in Ghana. Ghana Medical Journal, 52(4), 228. https://doi.org/10.4314/gmj.v52i4.10

Capewell, C., Ralph, S., \& Symonds, M. (2020). Listening to women's voices: Using an adapted photovoice methodology to access their emotional responses to diagnosis and treatment of breast cancer. Journal of Patient Experience, 7(6), 1316-1323. https://doi.org/10.1177/2374373520930463

Chang, D., Xu, H., Rebaza, A., Sharma, L., \& Dela Cruz, C. S. (2020). Protecting health-care workers from subclinical coronavirus infection. The Lancet. Respiratory Medicine, 8(3), e13. https://doi.org/10.1016/S2213-2600(20)30066-7

Clemente, M. R., Vieira, R., Martins, F., \& Andrade, A. I. (2014). Linguistic diversity in Aveiro, Portugal: Exploring linguistic landscape methodologies in the «Beira Mar» neighborhood. Internet Latent Corpus Journal, 3(1), 116-133. 
Costa, A. P., \& Amado, J. (2018). Análise de conteúdo suportada por software. Ludomedia

Costa, A. P., Loureiro, M. J., Reis, L. P., \& Neri de Souza, F. (2015). Análise de interações focada na colaboração e cooperação do modelo 4C. Revista Lusófona de Educação, 29, 19-39.

Fornari, L. F., \& Fonseca, R. M. G. S. da. (2020). Critical-emancipatory workshop analysis through qualitative analysis software. Qualitative Report, 25(13), 90-103.

Freitas, F., Ribeiro, J., Brandão, C., Souza, F. N. de, \& Costa, A. P. (2017). Experiência de utilizador em pacotes de software de análise qualitativa: da usabilidade à (auto)aprendizagem. Em A prática na Investigação Qualitativa: exemplos de estudos (1a parte) (Vol. 1, Número 1, pp. 147-172). Ludomedia.

Ganer N. (2016). Work related musculoskeletal disorders among healthcare professional and their preventive measure: a report. IJSRSET, 2(4), 693-8. https://doi.org/10.4103/00195278.146896

Gonçalves, J. P., Marques, C. G., \& Gonçalves, S. P. (2021). Manual de investigação qualitativa conceção, análise e aplicações. Pactor.

Liu S, Yang L, Zhang C, et al. (2020). Online mental health services in China during the COVID-19 outbreak. Lancet Psychiatry, (7), e17-18. https://doi.org/10.1016/S2215-0366(20)30077-8

Long, M. H., Bogossian, F. E. \& Johnston, V. (2013). The prevalence of work- related neck, shoulder, and upper back musculoskeletal disorders among midwives, nurses, and physicians: a systematic review. Workplace Health Saf, 61, 223-9. https://doi.org/10.1177/216507991306100506.

Luan, H. D., Hai, N. T., Xanh, P. T., Giang, H. T., Van Thuc, P., Hong, N. M., \& Khue, P. M. (2018). Musculoskeletal disorders: prevalence and associated factors among district hospital nurses in Haiphong, Vietnam. BioMed Research International, 2018. https://doi.org/10.1155/2018/3162564

Nascimento, T., \& Pestana, G. (2019). Improving efficiency in organizations by monitoring stress and promoting awareness and wellbeing at the workplace. European Conference on Knowledge Management, 2, 1197-1204. https://doi.org/10.34190/KM.19.165

Okuyucu, K., Gyi, D., Hignett, S., \& Doshani, A. (2019). Midwives are getting hurt: UK survey of the prevalence and risk factors for developing musculoskeletal symptoms. Midwifery, 79. https://doi.org/10.1016/j.midw.2019.102546

Okuyucu, K., Hignett, S., Gyi, D., \& Doshani, A. (2021). Midwives' thoughts about musculoskeletal disorders with an evaluation of working tasks. Applied Ergonomics, 90, 103263. https://doi.org/https://doi.org/10.1016/j.apergo.2020.103263

Presado, M. H., Marques, F. M., Baixinho, C. L., \& Cardoso, M. (2015). Lesões músculoesqueléticas nos enfermeiros especialistas em sáude materna. CIAIQ, 1, 193-198.

Rainbow, J. G., \& Steege, L. M. (2017). Presenteeism in nursing: An evolutionary concept analysis. Nursing Outlook. https://doi.org/10.1016/j.outlook.2017.03.005

Santos, M. A. F., Lopes, M. A. P., \& Botelho, M. A. R. (2018). Photovoice as a method of data collection in the study of motherhood over the age of 35: The power of images. Em A. P. Costa, L. P. Reis, F. N. de Souza, \& A. Moreira (Eds.), Advances in Intelligent Systems and Computing (Vol. 621, pp. 316-326). Springer International Publishing. https://doi.org/10.1007/978-3-319-61121-1_27

Serranheira, F., Cotrim, T., Rodrigues, V., Nunes, C., \& Sousa-Uva, A. (2012). Lesões musculosqueléticas ligadas ao trabalho em enfermeiros portugueses: "ossos do ofício» ou doenças relacionadas com o trabalho? Revista Portuguesa de Saúde Publica, 30(2), 193203. https://doi.org/10.1016/j.rpsp.2012.10.001

Taghinejad, H., Azadi, A., Suhrabi, Z. \& Sayedinia, M. (2016). Musculoskeletal disorders and their related risk factors among Iranian nurses. Biotech Health Sci, 3(1), e34473. https://doi.org/10.17795/bhs-34473.

Teixeira-Dias, J. J. C., Pedrosa de Jesus, H., de Souza, F. N., \& Watts, M. (2005). Teaching for quality learning in chemistry. International Journal of Science Education, 27(9), 1123-1137. https://doi.org/10.1080/09500690500102813 
Thinkhamrop, W., Sawaengdee, K., Tangcharoensathien, V., Theerawit, T., Laohasiriwong, W., Saengsuwan, J., \& Hurst, C. P. (2017). Burden of musculoskeletal disorders among registered nurses: evidence from the Thai nurse cohort study. BMC Nursing, 16(1), 68. https://doi.org/10.1186/s12912-017-0263-x

Topcu, G., Buchanan, H., Aubeeluck, A., \& Ülsever, H. (2020). Informal carers' experiences of caring for someone with Multiple Sclerosis: A photovoice investigation. British Journal of Health Psychology, n/a(n/a). https://doi.org/10.1111/bjhp.12482

Wang, C. C., Yi, W. K., Tao, Z. W., \& Carovano, K. (1998). Photovoice as a participatory health promotion strategy. Health Promotion International, 13(1), 75-86. https://doi.org/10.1093/heapro/13.1.75

Wang, C., \& Burris, M. A. (1997). Photovoice: Concept, methodology, and use for participatory needs assessment. Health Education \& Behavior, 24(3), 369-387. https://doi.org/10.1177/109019819702400309

Watts, M., \& Alsop, S. A. (2000). Terms of engagement: Learners and school science. Paper presented to the Annual Conference of the Canadian Society for the Study of Education.

Werremeyer, A., Skoy, E., Burns, W., \& Bach-Gorman, A. (2020). Photovoice as an intervention for college students living with mental illness: A pilot study. Mental Health Clinician, 10(4), 237243. https://doi.org/10.9740/mhc.2020.07.237 